\title{
Combination of Ropivacaine and Lidocaine for Long Lasting Locoregional Anesthesia
}

\author{
Lazăr Alexandra, Szederjesi J, Copotoiu Ruxandra, Copotoiu Sanda-Maria, Azamfirei L \\ Department of Anesthesia and Intensive Care, University of Medicine and Pharmacy, Tîrgu Mureș, Romania
}

\begin{abstract}
Objective: The aim of this study was to evaluate the clinical utility of Ropivacaine $0.5 \%$ and Lidocaine $0.5 \%$ anestethic combination in performing locoregional anesthesia, using either peripheral nerve stimulator or ultrasounds for brachial plexus block.

Study design: A prospective randomized clinical study was performed at the County Emergency Clinical Hospital of Tîrgu Mureș, between January and May 2013 on patients undergoing elective or emergency surgical interventions on upper limbs with locoregional anesthesia. Brachial plexus block with axillary approach was performed in 65 patients using randomly the nerve stimulator or the ultrasound guided technique. The parameters recorded were the duration of the anesthetic technique, the installation time and the length of anesthesia. All anesthetic incidents during and after anesthesia were observed as well. The recorded data were analyzed and statistically processed.

Results: We enrolled 40 (61.5\%) patients for the nerve stimulation technique and $25(38.5 \%)$ patients for ultrasound guidance. The quality of the block was acceptable, an inadequate anesthesia was reported in 9 patients (13.8\%). The mean time of installation of anesthesia was 34.36 ( \pm 11.56$)$ minutes, time recorded from the initiation of the anesthetic technique until complete motor block. The mean duration of the motor block was 481.3 ( \pm 128.6) minutes which represents over 8 hours. None of the patients required conversion of the anesthesia due to the extended period of the surgical intervention. One patient presented a mild allergic reaction to the anesthetic drugs.

Conclusion: Combination of the ropivacaine and lidocaine can be safely used for locoregional anesthesia, especially in those cases where long surgical intervention time is anticipated or in order to achieve a better postoperative analgesia.
\end{abstract}

Keywords: regional anesthesia, ropivacaine, lidocaine, brachial plexus, ultrasound

Received: 12 June 2013 / Accepted: 31 March 2014

\section{Introduction}

In upper limb surgery, the anesthetic choices comprise various techinques, locoregional anesthesia being one of the favourites, because of it's advantages over general anesthesia. Among these advantages the most important are the hemodynamic stability, the avoidance of airway manipulation or the usage of systemic analgetics (opioids) perioperatively.

In order to obtain a good quality anesthesia, the used anesthetic substances must offer a good anesthesia for a longer period of time. A good anesthesia is the anesthesia with a fast onset - to ease the patient's pain as fast as possible - and also the anesthesia which offers good surgical conditions and a long period of analgesia postoperatively.

The combination of Lidocaine and Ropivacaine meets these conditions. Lidocaine is an anesthetic with a fast onset, but with a shorter duration, while Ropivacaine has a slower onset, but a longer duration of anesthesia. They can be combined in order to obtain a good quality anesthesia.

The aim of this study is to evaluate the clinical utility of the anesthetic combination Ropivacaine and Lidocaine in performing locoregional anesthesia, brachial plexus block by axillary approach, using either neurostimulation or the ultrasound-guided method.

\section{Material and method}

We carried out a prospective, randomized study in the County Emergency Clinical Hospital of Tîrgu Mureș, between February and March 2013. The study obtained the approval of the Ethics Committee of the County Emergency Clinical Hospital of Tîrgu Mureș (No. 2987/2013) and also of the Ethics Committee of the University of Medicine and Pharmacy of Tîrgu Mureș (No. 40/2013).

The study enrolled adult patients, with emergency or elective surgery on the upper limbs. The performed anesthesia was brachial plexus block by axillary approach and using the multiple injection technique.

\section{Materials}

- Peripheral neurostimulator - Stimuplex HNS 12 (B Braun, Melsungen, Germany);

- Ultrasound G\&E Logiq- linear probe 9 (General Electric,USA);

- Stimulation needles of $50 \mathrm{~mm}$ in length with a 30 degrees cut of bevel;

- Hypoechoic stimulating needle, Ultraflex by BBraun.

In order to obtain a combination of Ropivacaine $0.5 \%$ with Lidocaine $0.5 \%$, we combined $10 \mathrm{ml}$ of Ropivacaine $1 \%$ with $10 \mathrm{ml}$ of Lidocaine. The anesthetized nerves were the musculocutaneus, radial, median and the ulnar nerve. The quantity of anesthetic for each nerve varied accordingly to the length of the surgical intervention. 


\section{Study protocol}

After informing the patient and obtaining a written consent, the patient was positioned supine with the arm of the injured limb at a 90-degree angle.

After assessing a venous access and monitoring the vital signs (non-invasive blood pressure, three leads ECG, peripheral oxygen saturation), the puncture site was established by palpation of the axillary artery. Subcutaneous anesthesia was performed using $1-2 \mathrm{ml}$ of Ropivacaine $0.5 \%$ and Lidocaine $0.5 \%$. The anesthetic technique was performed using either the peripheral neurostimulator or ultrasound guidance.

When the peripheral neurostimulation method was used, the $50 \mathrm{~mm}$ needle position relative to the axillary artery was anteriorly for the musculocutaneus and the median nerve, anterior-inferior for the ulnar nerve and posterior-inferior for the radial nerve. The stimulation parameter used at the beginning of the procedure was of $1.2 \mathrm{~mA}$ and when the characteristic motor response was obtained, the amplitude of the stimulation was gradually reduced, the anesthetic infusion being made at $\leq 0.5 \mathrm{~mA}$.

The ultrasound-guided method implied spotting the axillary artery and the surrounding nerves by direct visualization. For the brachial plexus nerves localization a $50 \mathrm{~mm}$ hypoechoic needle was used and as an adjuvant method a peripheral neurostimulator with low amplitude impulses (0.2-0.4 mA) was used.

The technique used for anesthetic administration was the triple injection technique, the anesthetic volume administered ranged from 4 to $10 \mathrm{ml}$ for each nerve equivalent to 20-50 mg for each anesthetized nerve.

A successful anesthesia was considered when we obtained a complete sensorial and motor block. In case of an unsuccessful anesthesia, we identified the territory and supplemented the anesthesia by a new puncture, or local anesthesia with Lidocaine $1 \%$ was delivered.

The quality of postoperative anesthesia was assessed by reevaluation of motor and sensory block quality and quantification of systemic analgesics need.

A specific data sheet was used for data collection, which included the following:

- the patient $s$ identification code - number of the observation chart;

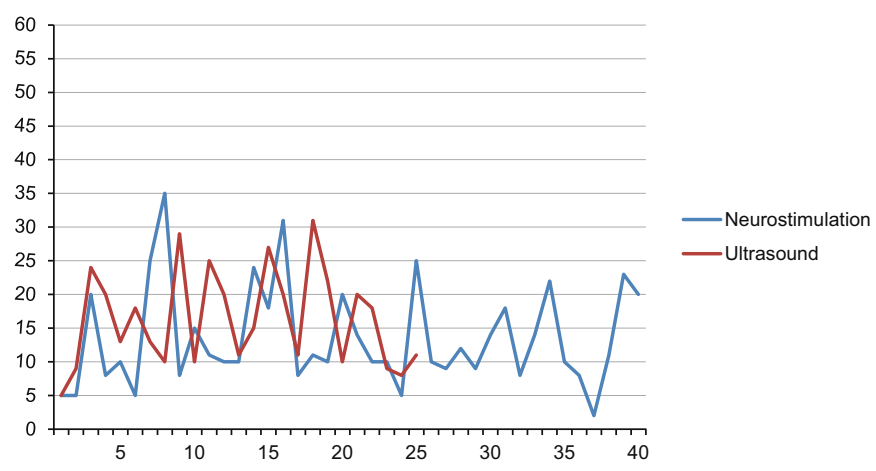

Fig. 1. Performing time, $p=0.1453$
- the primary diagnosis and also the secondary diagnoses;

- anthropometric data, such as age, height, weight;

- the method by which the anesthesia was performed neurostimulation or ultrasound-guided;

- the anesthetized nerves;

- the quantity of anesthetic administered for each nerve;

- when the neurostimulation method was used the minimum amplitude of neurostimulation was recorded;

- the exact time of anesthesia initiation;

- the exact time of anesthesia finalization;

- the exact time of motor/sensory block installation;

- during anesthesia, intraoperative and postoperative incidents and accidents were registered.

The processed data were:

- anesthesia installation time - the time recorded from the moment of anesthesia initiation until the moment of obtaining complete motor/sensory block.

- the testing methods were the heat sensitivity and response to painful stimuli;

- total duration of anesthesia-the period between the initiation of the anesthesia and the moment when the patient felt the first pain sensation or systemic analgesics was needed.

Incidents and accidents recorded were:

- during anesthesia - arterial punctures, allergic reactions;

- intraoperative - necessity of anesthesia supplementation by the surgeon, with local anesthetics;

- postoperative - long term reactions to the anesthetics

For data processing we used Microsoft Excel (by Microsoft, USA) and Graph Prism 6.0 (by Graph Pad Prism Inc. USA) as statistical processing data programs.

\section{Results}

The study enrolled 65 patients aged between $17-84$ years, 9 females and 56 males.

The patients were randomly divided into two groups, the peripheral neurostimulation group (NG) enrolled 40 patients and the ultrasound-guided group (UG) 25 patients.

The processed data were unpaired, numerical data. The collected data passed the Kolmogorov Smirnov normality test and we assumed a Gaussian distribution.

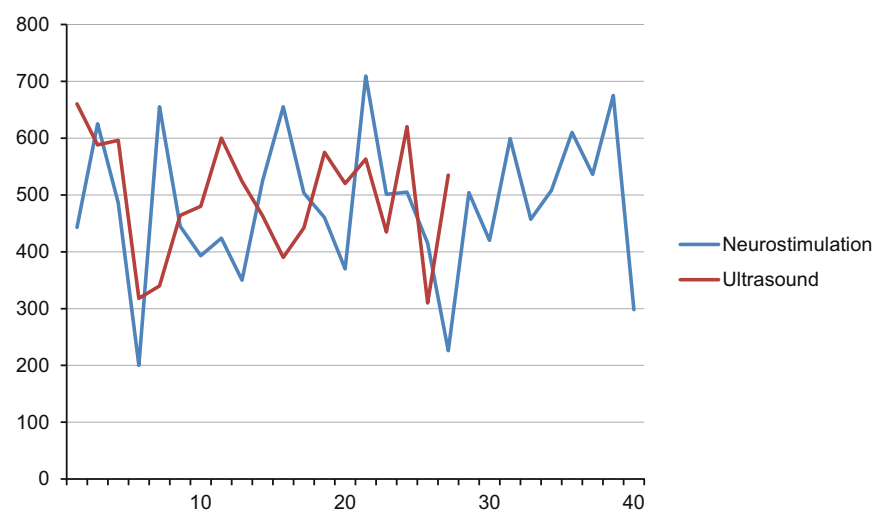

Fig. 2. Total duration of anesthesia, $p=0.6954$ 
The mean time of anesthesia installation was 34.138 $( \pm 11.807)$ minutes. No statistically significant differences were observed between the two groups, $\mathrm{p}=0.3293$ (Figure $1)$.

The mean duration of anesthesia was $487.66( \pm 117.69)$ minutes with a minimum of 200 minutes and a maximum of 709 minutes. No statistically significant differences were observed between the two groups, $\mathrm{p}=0.6954$ (Figure 2).

None of the enrolled patients required conversion of anesthesia due to prolonged surgical intervention time or due to insufficient anesthesia.

A single mild allergic reaction was recorded and in 9 cases $(13.8 \%)$ the anesthesia was inadequate.

\section{Discussion}

The aim of this study was to evaluate the efficiency of the anesthetics combination Ropivacaine and Lidocaine, equivalent concentrations, in respect of time of anesthesia installation and also of the quality and duration of brachial plexus block anesthesia performed with the help of these anesthetics.

The reason for choosing this combination was to study the anesthetics combined effects the purpose being obtaining an anesthesia with a longer duration longer and with a shorter installation period.

Mixtures of some anesthetics with a shorter induction time (Lidocaine) and a longer acting agent (Ropivacaine) are frequently used in order to obtain a short installation time for the sensory as well as for the motor block [1], and also a longer postoperative analgesia and anesthesia [2].

Ropivacaine is a long acting anesthetic, part of the amino-amide class. It is the pure isolates $\mathrm{S}$-enantiomer of the substance introduced to reduce the toxicity of local anesthetics and to improve the quality of motor and sensory block [3]. This anesthetic is widely used especially for its low toxicity on the central nervous system and also on the cardiovascular system [4].

Lidocaine was the first amino-amide local anesthetic, being introduced in 1948. It provides a short induction time, but the high potential of neurotoxicity limits its usage to shorter duration interventions [5]. Its mechanism of action relies on the depression of neuronal excitability via the blockage of voltage dependent $\mathrm{Na}$ channels in the cell membrane. By this mechanism the antiarrhythmic and local anesthetic effects of Lidocaine are being produced, but also this is the same mechanism of its neurotoxic effect [6].
For a long duration anesthesia with a short installation time, the combination of Lidocaine and Ropivacaine is one of the most appropriate, conclusion drawn also by Fanelli et al [7].

Our study results can be related also with the results obtained by Curvillon Pet al. regarding the installation time of the anesthesia performed using this combination of anesthetics [8], but the literature is poor in studies on this exact combination of anesthetics.

From our results it can be noticed the shorter anesthesia installation time and the fact that we obtained a total duration of anesthesia and analgesia for over 8 hours present an important advantage with benefits regarding the quality of the anesthetic act and also on patient satisfaction.

Regarding the adverse reaction upon using this combination of anesthetics, the fact that we encountered just one mild allergic reaction suggests that this combination has a high degree of safety.

\section{Conclusions}

Ropivacaine $0.5 \%$ in combination with Lidocaine $0.5 \%$ constitutes a local anesthetic with a higher degree of safety and a lower number of unsuccessful anesthesia. This combination proved its effectiveness by offering a short induction time anesthesia with a prolonged postoperative anesthesia and analgesia.

\section{References}

1. Cuvillon P, Nouvellon E, Ripart J, Boyer JC, Dehour L, Mahamat A, et al. A comparison of the pharmacodynamics and pharmacokinetics of bupivacaine, ropivacaine (with epinephrine) and their equal volume mixtures with lidocaine used for femoral and sciatic nerve blocks: a double-blind randomized study. Anesth Analg. 2009;108:641-649.

2. Ye F, Feng $Y X$, Lin JJ. A ropivacaine-lidocaine combination for caudal blockade in haemorrhoidectomy. J Int Med Res 2007;35:307-313.

3. Hansen TG. Ropivacaine: A pharmacological review. Expert Rev Neurother. 2004;4:781-91.

4. Zink W, Graf BM. The toxicity of local anesthetics: the place of ropivacaine and levobupivacaine. Curr Opin Anaesthesiol. 2008;21:645-650.

5. Feldman HS, Covino BG. Comparative motor-blocking effects of bupivacaine and ropivacaine: A new amino amide local anaesthetic, in the rat and dog. Anaesthesia and Analgesia. 1991;73:373-84.

6. Walker IA, Slovis CM. Lidocaine in the treatment of status epilepticus. Acad Emerg Med. 1997;4:918-22.

7. Fanelli G, Casati A, Beccaria P, et al. A double blind comparison of ropivacaine, bupivacaine, and mepivacaine during sciatic anf femoral nerve blockade. Anesth Analg. 1998;87:597-600.

8. Cuvillon $P$, Nouvellon E, Ripart J, et al. A comparison of the pharmacodynamics and pharmacokinetics of bupivacaine, ropivacaine (with epinephrine) and their equal volume mixtures with lidocaine used for femoral and sciatic nerve blocks: a double-blind randomized study. Anesth Analg. 2009;108:641-649. 